\title{
Design and Development of a Digital Archive of Illuminated Manuscripts
}

\author{
Maristella Agosti, Nicola Ferro, Nicola Orio \\ Department of Information Engineering \\ University of Padua, \\ Via Gradenigo, 6/a - 35131 Padova - Italy \\ \{agosti, ferro, orio\}@ dei.unipd.it
}

\begin{abstract}
The aim of this paper is to report the research results of an ongoing project that deals with the exploitation of a digital archive of drawings and illustrations of historic documents for research and educational purposes. According to the results on a study of user requirements, we have designed tools to provide researchers with innovative ways for accessing the digital manuscripts, sharing, and transferring knowledge in a collaborative environment. We have found that the results of scientific research on the relationships between images of manuscripts produced over the centuries can be rendered explicit by using annotations. The methodology has been implemented by a prototype system that is currently used by researchers in the field of illuminated manuscripts.
\end{abstract}

\section{Introduction}

The ideas and concepts reported in this paper build upon our experience on the analysis of the user-requirements, the design of a methodology, the development of a prototype system, and the analysis of the feedback from real users of a digital archive of historical material. The archive aims at the study and research on illuminated manuscripts, which are books, usually handwritten, that include illustrations and, in the past centuries, were manually and artistically decorated. Illuminated manuscripts are still the subject of scientific research in different areas, namely history of arts and history of science, and all the disciplines that are related to the subject of the book - e.g., botany, astronomy, medicine [5]. Before the invention of photography, illuminated manuscripts have been the main mean for the dissemination of the scientific culture, and to this end play a major role as witness of the cultural heritage of different cultures, in Europe, Asia, and in the countries under the influence of the Arabic culture.

The prototype digital archive of illuminated manuscripts that has been developed within our research activities has been called Ipsa, which stands for Imaginum Patavinae Scientiae Archivum (archive of images of the Paduan science) $[3,2,4]$, because the main focus of our initial project was to provide a tool for the analysis of the role of the Paduan school during the Middle Ages and the Renaissance for the spread of the new scientific method in difference sciences, from medicine to astronomy. IPSA is a case study for our research on methodologies and tools for researchers and scholars working on the study, the preservation and the dissemination of the cultural heritage.

We participate in a previous research project regarding the feasibility study of a digital archive for the preservation of the cultural heritage [1]. The feasibility study, called ADMV which stands for Archivio Digitale della Musica Veneta (digital archive of the Venetian music), regarded the creation of a digital archive of images of manuscripts of musical scores, digitized versions in MIDI format, and recordings of performances of musical works by Venetian composers, as Marcello and Pellestrina. The feasibility study was the motivation for a subsequent research project aimed at developing novel ways of indexing and retrieving musical documents in a digital library, both in symbolic and in audio forms [7].

The paper is divided in two parts. The first part describes the particular requirements that have been collected through continuous interaction with the researchers on illuminated manuscripts who will be the main users of a digital archive. The second part describes the main functionalities of the prototype system that has been developed accordingly with the user requirements.

\section{Requirements for the Design}

The development of models and tools for researchers and scholars in the area of illuminated manuscripts requires a careful analysis of user requirements [6]. It is likely that the requirements for carrying out scientific research will be more complex and articulated than requirements for com- 
mon users. Common users access an image digital archive to acquire information in a given field, researchers access the archive to disclose knowledge and discover new relationships among digital objects.

Research users have been interviewed in a number of meetings, which involved art historians, historians of science, botanists, and astronomers. The first ideas about users requirements have been formalized in a draft proposal that has been presented and discussed with research users. Refinements of the proposal have been added, by reiterating the points of the meetings and the discussions.

\subsection{Highly Dynamic Documents Records}

Almost every digital archive dynamically changes over the years, mainly because of new acquisitions that increase the number of documents. This is also true for a digital archive of illuminated manuscripts, but there are other reasons that produce changes on the archive over time. The creation of records describing the documents and the images in a illuminated manuscript, as for any collection of historical works, is part of the scientific research itself. Some examples of changes to records are, for instance, that new relationships with other works have been discovered, or that the attribution to a given author became less certain.

These examples of possible changes make a collection of illuminated manuscripts more dynamic than, for instance, a collection of printed books. Moreover, unlike bibliographic records, there is no universal agreement on a fixed standardized description of objects of the type of hand-drawings; apart from the name of the subjects of the drawing, a complete record should include the style, the way colors are used, the size of the images, to cite only a few features. Scientific research on illuminated manuscripts aims also at defining standards for the descriptions of the documents.

Since creating a new record or modifying an existing one is part of the scientific work of researchers, the data management has to deal with intellectual rights. A researcher may prefer that some of the newly created records are not accessible by other users, at least until the results of his research have been published and his work have been acknowledged. This situation implies that users may decide which information can be shared with other users and which can not.

\subsection{A Collaborative Environment}

The study of illuminated manuscripts involves a number of researchers from different fields. In fact, illuminated manuscripts are of interest for both the historian of art and the historian of science, but at the same time, a herbal is of interest for the botanist because they represent plants and their possible variations through the centuries, a codex is useful for researchers on the evolution of civil and penal laws, an astrological book may give insights to researchers in medicine on the way stars where perceived to influence the health of people and to astronomers on how constellations where seen and represented. Hence, the scientific research on illuminated manuscripts involves a number of persons with different expertise, which should be able to cooperate in order to share their different knowledge and background.

A digital archive of illuminated manuscripts has to provide a collaborative environment, such as in [8], where researchers should be able to interact and give different contributions on the definitions and redefinitions of objects in the manuscript. For this reason, it is necessary to consider different levels of users of the archive. Apart from the administrators, the group of research users should be able to modify the records of the underlying database when new features of the stored objects are discovered.

\subsection{Evolutions of Manuscripts}

During the Middle Age the primary role of illuminated manuscripts as a tool for scientists was lost, because authors of drawings were more interested on aesthetics than on realism. For this reason, images were no more created looking directly at real objects, but they were copied from or inspired by images of existing manuscripts. The resulting drawings became increasingly different from the subjects they should represent, because each author added new modifications on parts of the objects depending on personal stylistic choices.

For researchers, it is of primary importance to state if drawings of a illuminated manuscripts are copied from previous manuscripts or if they are directly inspired by the nature. The disclosure of a link between two images belonging to two independent manuscripts, because one was the source for creating the other, allows to draw connections between the art of natural representations during the years and across the countries. On the other hand, the proof that two images have been independently created, notwithstanding their apparent resemblance, because for instance the authors could not have been in contact, allows to refine the map of existing connections among different manuscripts.

The use of realistic images in illuminated manuscripts come back to life since the XIVth century and further developed during Renaissance. Among its goals, Ipsa aims at helping researchers investigating the role played by the University of Padua in the creation of this modern realistic and exact iconography. The disclosure that the images of a manuscripts have been created as realistic copies of objects witnesses the emerging role of the scientific culture. The disclosure of a relationship between the style of images of different manuscripts, even if both are realistic representa- 
tion of nature, helps tracking the spread of the new scientific culture over Europe.

\subsection{Disclosure of New Knowledge}

One of the most important aims of the research on illuminated manuscripts of scientific illustration is the disclosure of hidden relationships between illustrations created by different authors. In particular, it is of primary importance for researchers to discover if illustrations have been copied from images of other manuscripts, if they have been merely inspired by previous works, or if they are directly inspired by nature. The disclosure of such a relationship between two images belonging to two manuscripts allows researchers to draw connections between the art of scientific illustration through the years and among different countries.

A major user requirement concerns the possibility of enriching the digital archive by highlighting explicit relationships that have been discovered by a researcher. In particular, a research user should be able to create links that connect one image to another that it is related to, in some way. It is important to keep in mind that images belong to different manuscripts and that their relationship may not be so obvious. Each disclosure of a relationship adds new information to the archive content, which should be shared with collaborators and students. The analysis of user requirements on link management has brought to light a number of advisable features that could be implemented.

- Link authorship: The creation of a link between two or more images depends on the scientific results of a researcher, who owns the intellectual rights to the disclosure of a new relationship between images; for this reason the author of each new link has to be recorded by the system.

- Link typology: Since two images can be related for a number of different reasons, the kind of relationship should be explicit. Different typologies of links are envisaged to express the possibility that an image is the progenitor of a set of other images, or that two images are a copy of one another, and so on.

- Paths: Links may form historical paths among images, because images in a manuscript can be copies of another one which in turn are copies themselves of previous illustrations; hence two images may not be directly linked, because there is no direct relationship between them, but it could be possible to follow a path from one to the other by exploiting existing links.

We propose to represent these relationships through an annotation system that is built over an existing image digital archive. The main service provided by the annotation system consisting of a set of tools for image annotation. Given the particular requirements, a special focus is given to annotations that connect, or make a reference, from one image to another in the archive. We refer to this kind of annotations as linking annotations. There are a number of different reasons that may point out a relationship between two images, depending on historical, aesthetical, and technical considerations. For this reason, we proposed a taxonomy for linking annotations [2], which is divided in two classes, including annotations that express either hierarchical or relatedness links.

\subsection{Presentation of Digital Images}

A digital archive of illuminated manuscripts has the double role of preserving the cultural heritage and giving access to users in a networked environment. As it always happens in this situation, there is a trade-off between the high quality required for preservation and the small size needed for transfer over the network. Moreover, it has to be considered that research users should be able to perform comparisons among images belonging to different manuscripts that, in principle, may differ in their original size. According to research users, the number of images that should be presented on the computer screen varies from one to a maximum of six.

This last requirement implies that the image size, and hence its resolution, can dynamically vary depending on the context, because in principle a link can be created between any couple of images. The transfer load can be reduced through the use of thumbnails, at least for the first presentation of images. It can be noted that thumbnails can be also a viable solution when the comparison between images is not part of the scientific research but it can be used for dissemination to students or, if future releases of Ipsa will be available on the Web, to casual users without a controlled access.

Image acquisition is another important issue, because researchers should be able to analyze also small details of images. On the other hand, researches needs also to see the image of the complete page of a manuscript, because it gives the context in which a particular object is presented. Moreover, many manuscripts have more than a single image for each page, with images surrounding or overlapping with text. For these reasons, it is advisable to carry out multiple acquisitions of the same page, with different resolutions depending on the level of detail needed for the analysis by researchers.

\subsection{Personalised Users' Views}

Linking annotations add an hypertextual structure to the archive, which is different for each of the users and reflects his personal knowledge on the field, as shown in figure 1 


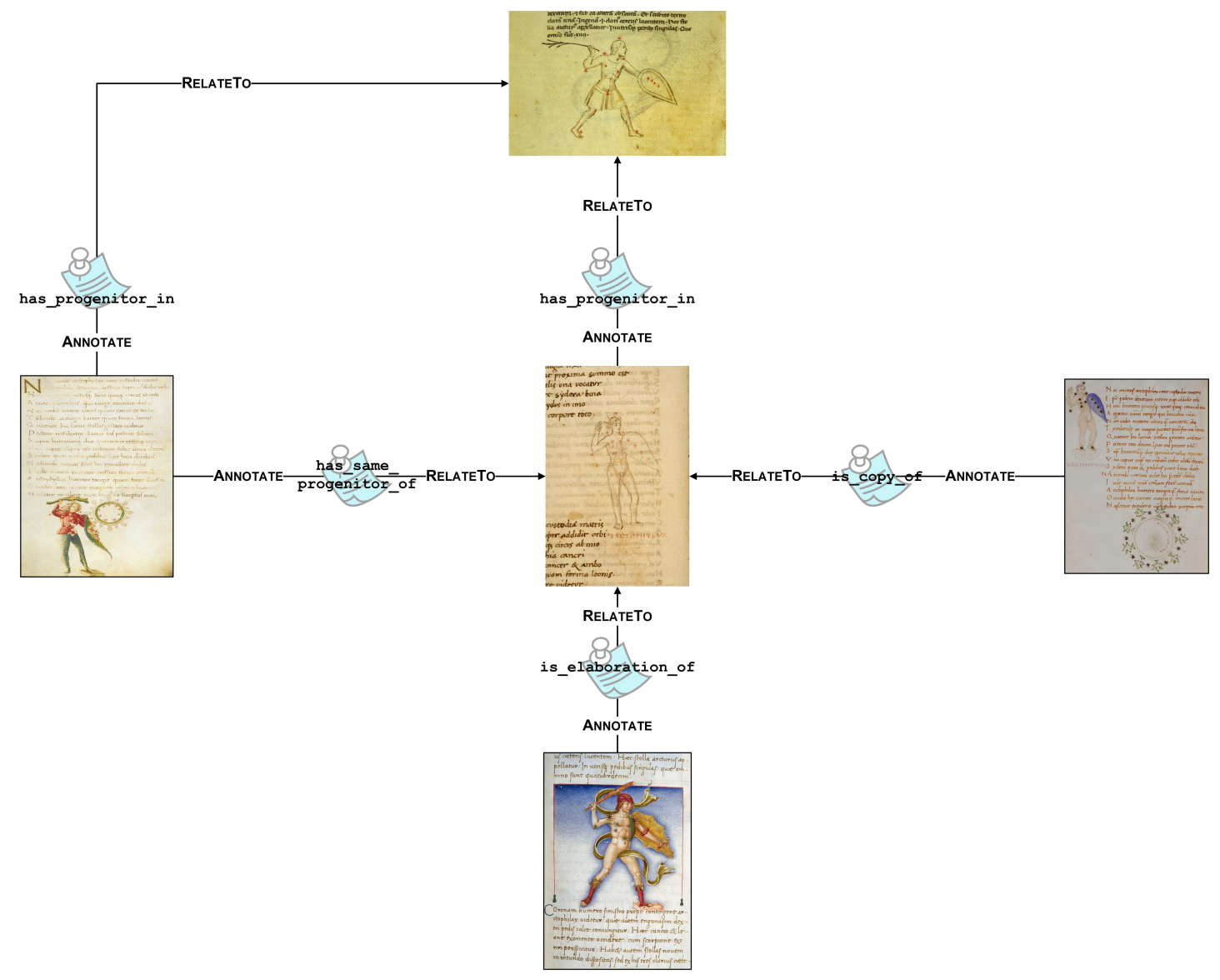

Figure 1. Example of a personalized view on some linked images.

If a mechanism of information sharing for collaborative environments is provided, researchers may cooperate in their study on illuminated manuscripts by sharing their knowledge on the domain. Users may benefit from the visualisation of both hierarchical and relatedness relationships among images.

\section{IPSA Prototype}

A prototype implementing the proposed methodology has been developed. Figure 2 shows the main page of the IPSA prototype, where the functionalities offered to the user are highlighted.

Figure 3 shows the search page of the IPSA prototype. The user can add and select multiple fields to specify his query: in the example, the author of the manuscript and the names of objects that appear in the manuscript.

Figure 4 shows results of the search specified in figure 4 .

Figure 5 shows the selection of an image from the results: information and metadata about the image are reported; the image can be explored and navigated; the annotations linking the image to other images are displayed.

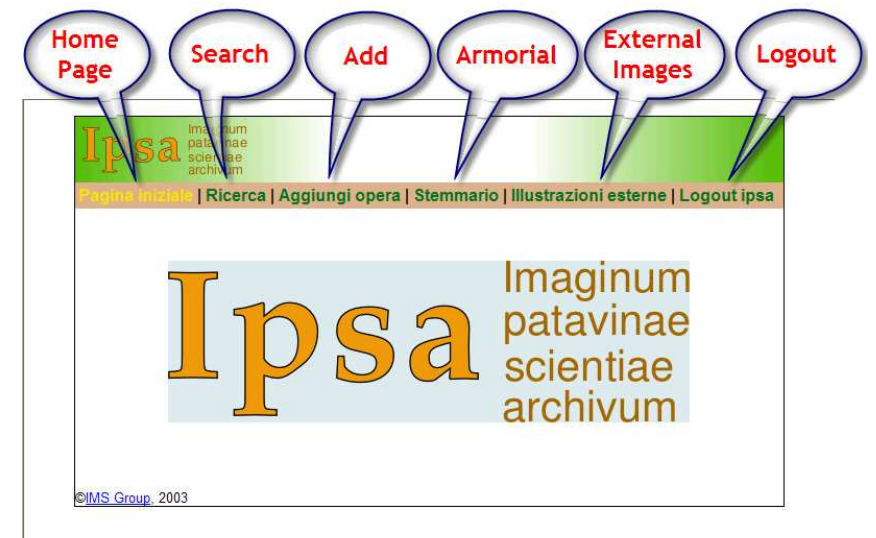

Figure 2. Main page.

Finally, figure 6 shows the interface for exploring and inspecting an image. 


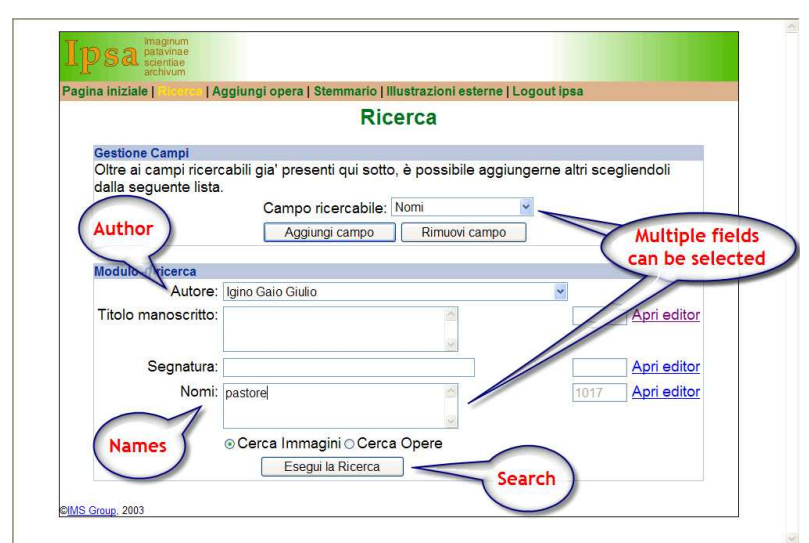

Figure 3. Search page.

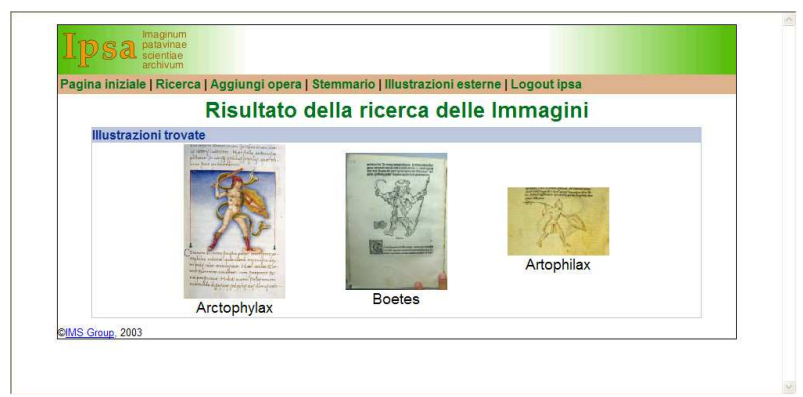

Figure 4. Search results.

\section{Conclusions}

A mechanism for linking annotations is proposed as a tool for researchers working in a collaborative environment. In particular, linking annotations can be a valuable tool for describing the evolution of scientific illustrations over the years. The proposed approach can be extended also to other application domains, in particular in the field of preservation and dissemination of cultural heritage.

\section{Acknowledgements}

The work reported in this paper was partially funded by Italian Ministry of Education (MIUR) under the Project of Relevant National Interest (PRIN) called "Methods for a digital corpus of illuminated manuscripts". The work was also partially supported by the DELOS Network of Excellence on Digital Libraries, as part of the Information Society Technologies (IST) Program of the European Commission (Contract G038-507618).

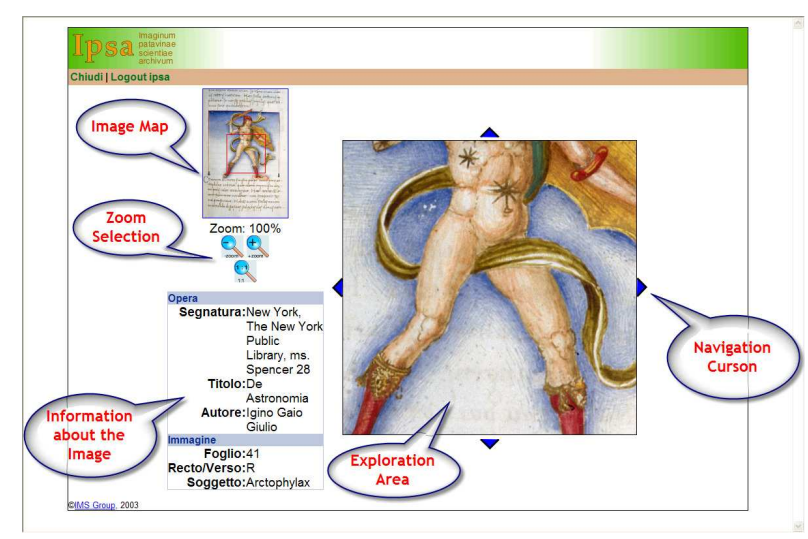

Figure 6. Image exploration capabilities.

\section{References}

[1] M. Agosti, F. Bombi, M. Melucci, and G. Mian. Towards a digital library for the Venetian music of the eighteenth century. In DRH 98: Selected papers from Digital Resources for the Humanities, pages 1-16. Office for Humanities Communication, 2000.

[2] M. Agosti, N. Ferro, and N. Orio. Annotating Illuminated Manuscripts: an Effective Tool for Research and Education. In Proc. 5th ACM/IEEE-CS Joint Conference on Digital Libraries (JCDL 2005), pages 121-130. ACM Press, New York, USA, 2005.

[3] M. Agosti, N. Ferro, and N. Orio. Annotations as a Support to Research Users. In Proc. 7th International Workshop of the EU Network of Excellence DELOS on Audio-Visual Content and Information Visualization in Digital Libraries (AVIVDiLib'05), pages 117-120. Centromedia, Viareggio, Italy, 2005.

[4] M. Agosti, N. Ferro, and N. Orio. Graph-based Automatic Suggestion of Relationships among Images of Illuminated Manuscripts. In Proc. 21 st ACM Symposium on Applied Computing (SAC 2006), pages 1063-1067. ACM Press, New York, USA, 2006.

[5] M. Canova. Hyginus De Astronomia. In The Splendor of the Word. Medieval and Renaissance Illuminated Manuscripts at the New York Public Library, pages 337-339). Harvey Miller, New York, USA, 2006.

[6] G. Crane. Cultural heritage digital libraries: Needs and components. In Proc. 6th European Conference on Research and Advanced Technology for Digital Libraries (ECDL 2002), pages 626637. LNCS 2458, Springer, Heidelberg, Germany, 2002.

[7] M. Melucci and N. Orio. Combining melody processing and information retrieval techniques: Methodology, evaluation, and system implementation. Journal of the American Society for Information Science and Technology, 55(12):1058-1066, October 2004.

[8] U. Thiel, H. Brocks, I. Frommholz, A. Dirsch-Weigand, J. Keiper, A. Stein, and E. J. Neuhold. COLLATE A collaboratory supporting research on historic European films. International Journal on Digital Libraries, 4(1):8-12, August 2004. 


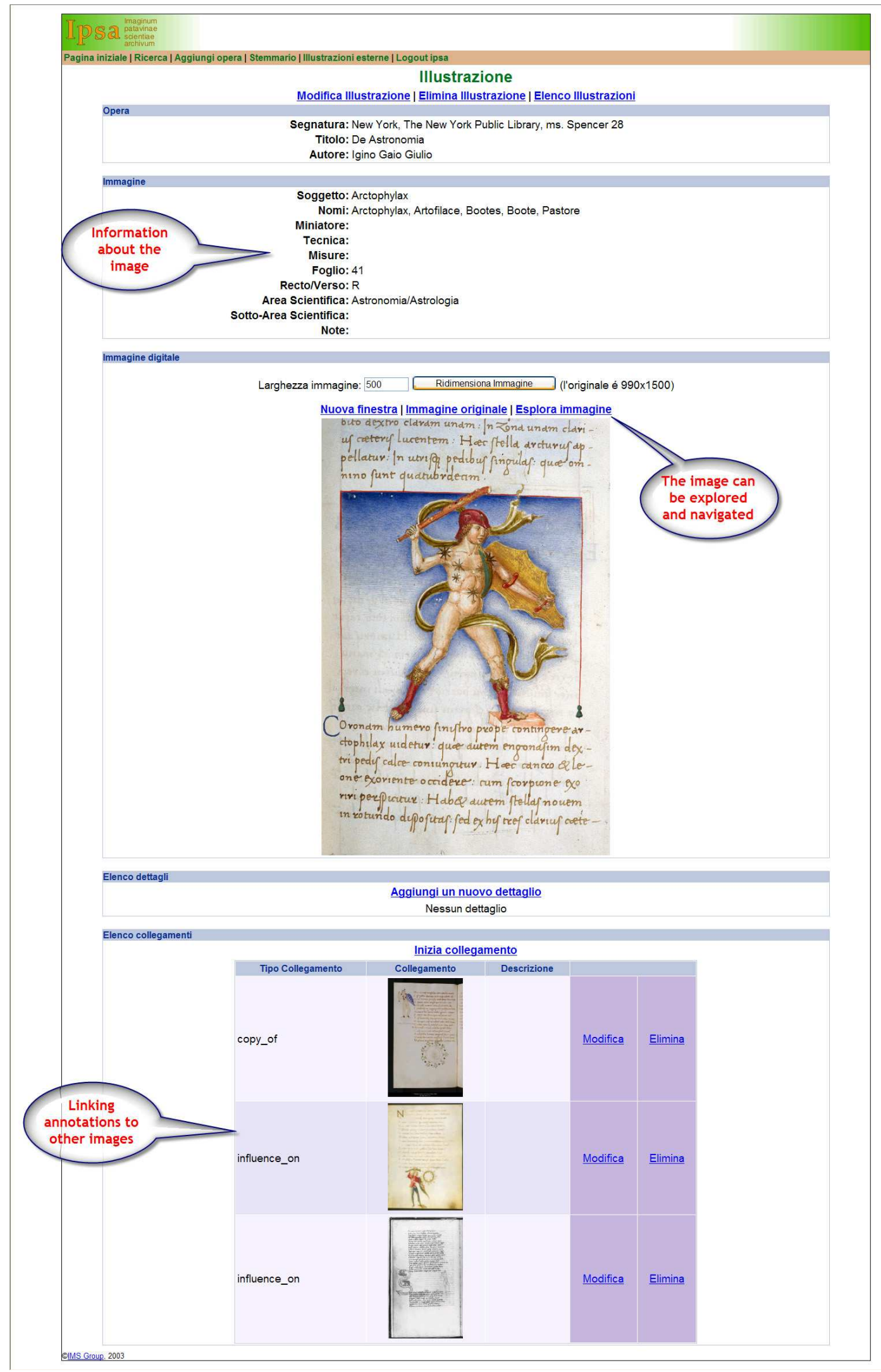

Figure 5. Visualization of an image and its annotations. 